\title{
Modeling of fluid flow in periodic cell with porous cylinder using a boundary element method
}

\author{
R.F. Mardanov ${ }^{\mathrm{a}}$, S.J.Dunnett ${ }^{\mathrm{b}}$, S.K. Zaripov ${ }^{\mathrm{a}}$ \\ ${ }^{a}$ Kazan Federal University, Kazan, Russia \\ ${ }^{b}$ Loughborough University, Loughborough, UK
}

\begin{abstract}
The problem of viscous incompressible flow past a periodic array of porous cylinders (a model of flow in an aerosol filter) is solved. The approximate periodic cell model of Kuwabara is used to formulate the fluid flow problem. The Stokes flow model is then adopted to model the flow outside the cylinder and the Darcy law of drag is applied to find the filtration velocity field inside the porous cylinder. The boundary value problems for biharmonic and Laplace equations for stream functions outside and inside the porous cylinder are solved using a boundary elements method. A good agreement of numerical and analytical models is shown. The analytical formulas for the integrals in the expressions for the stream function, vorticity and Cartesian velocity components are obtained. It is shown that use of analytical integration gives considerable advantage in computing time.
\end{abstract}

Keywords: porous cylinder, Stokes flow, Darcy model, boundary element method

\section{Introduction}

The problem of air flow through porous bodies has practical applications for many different environmental problems, for example air cleaning devices aerosol filters, wire screens, aerosol respirators. It is known that the use of

Email address: S.J.Dunnett@lboro.ac.uk (S.J.Dunnett) 
5 porous cylinders as elements of aerosol filters significantly increases the efficiency of the deposition of aerosol particles [1, 2]. Also, during the filtration process the suspended particles, deposited on fibers, form a porous layer. The distortion of the fluid flow through the filter elements covered by the porous layers considerably affects the inertial and diffusional capture of further aerosol 10 particles [3]. Hence it is important that efficient mathematical models of fluid flow past porous bodies are developed in order to calculate the two-phase flows of dusty air.

In the work presented here the fluid flow in a periodic array of porous cylinders, modelling the air flow in an aerosol filter, is considered under the assump15 tion of viscous incompressible flow. The approximate periodic cell model of Kuwabara [4] is used to formulate the fluid flow problem. The cell model with Kuwabara boundary conditions was used previously by Stechkina [5], Kirsh [1] to determine the velocity field of the flow over a porous cylinder in the case of small Reynolds number flow, using analytical solution and the collocation 20 method. In this work the Stokes flow model is adopted outside the cylinder in the cell and inside the porous cylinder the Darcy law of drag is applied to find the filtration velocity field. The resulting boundary value problems for the biharmonic and Laplace equations for the stream function and pressure, with boundary conditions on the porous surface, are formulated. The boundary elements method (BEM) is then used to solve the considered boundary value problems. The analytical formulas for the integrals in the expressions for stream function, vorticity and Cartesian velocity components are found. The numerical solution is compared with analytical solution obtained with Kuwabara boundary conditions. domain and fluid flow between eccentric cylinders were also solved by the method developed in this paper and results obtained. 


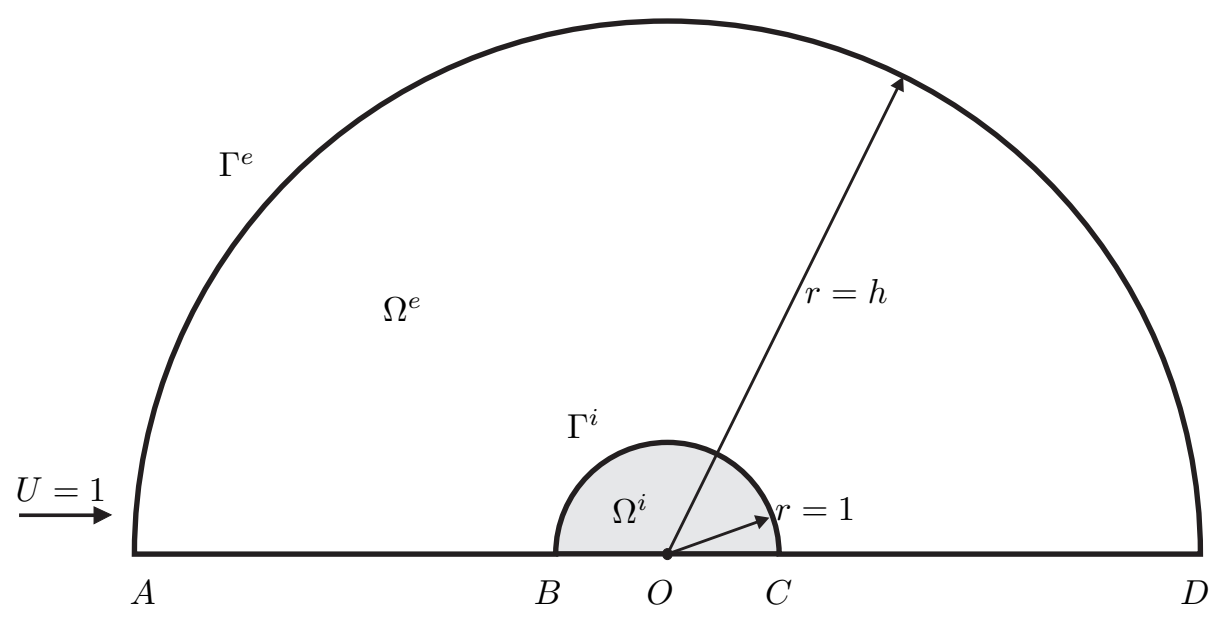

Figure 1: Fluid flow domain

\section{Problem statement}

The two-dimensional flow of an incompressible gas in a periodic cell, of 35 radius $h$, with a porous cylinder of radius $R_{c}$ at a small Reynolds numbers is considered. The radius $R_{c}$ and the fluid velocity $U$, at the cell boundary, are used as length and velocity scales. Due to the fluid flow symmetry we select as a calculation domain the upper part of the periodic cell.

The outer fluid domain $\Omega^{e}$ is the half annulus region formed by the cell

40 boundary $r=h=1 / \sqrt{\alpha}(\alpha=1-\varepsilon, \varepsilon-$ porosity $)$ and the boundary of the cylinder, $r=1$. The porous zone $\Omega^{i}$ is the inner half-circle of unity radius (fig.(1). The problem of fluid flow in domain $\Omega^{e}$, assuming Stokes flow, can be reduced to the solution of the biharmonic equation for the stream function $\psi^{e}(x, y)$

$$
\Delta^{2} \psi^{e}=0
$$

with the conditions on the boundary $\Gamma^{e}$ of the domain $\Omega^{e}$. On the outer bound45 ary $A D$ we have

$$
\psi^{e}=y, \quad \omega^{e}=0,
$$

where $\omega^{e}(x, y)=-\Delta \psi^{e}$ is the vorticity. Condition (2) is valid for small values of $\alpha$ as shown by Marshall et al [6] who obtained accurate results for fluid 
flow in a filter for $\alpha<0.2$. On the symmetry lines $A B$ and $C D$ the following conditions are applied:

$$
\psi^{e}=0, \quad \omega^{e}=0 .
$$

The stream function $\psi^{i}(x, y)$ in the porous zone $\Omega^{i}$ with the boundary $\Gamma^{i}$, assuming the Darcy law for fluid flow, is described by the Laplace equation

$$
\Delta \psi^{i}=0
$$

with the symmetry condition along the line $B O C$

$$
\psi^{i}=0 .
$$

On the common boundary $B C$ of the domains $\Omega^{e}$ and $\Omega^{i}$ the conditions of equality of the pressure and flow rates

$$
\begin{aligned}
& p^{e}=p^{i}, \\
& \psi^{e}=\psi^{i}
\end{aligned}
$$

as well as the condition proposed by Beavers and Joseph [7] are applied. The condition of Beavers and Joseph gives a linear relationship between the normal derivative of the slip velocity and the difference of the components of velocities $u_{\theta}^{e}$ and $u_{\theta}^{i}$ of outer and interior flows:

$$
\frac{\partial u_{\theta}^{e}}{\partial r}=-\alpha_{s} S\left(u_{\theta}^{i}-u_{\theta}^{e}\right),
$$

${ }_{60}$ where $\alpha_{s}$ is the dimensionless coefficient (the slip coefficient), $S=R_{c} / \sqrt{k}, k$ is the porous medium permeability. The quantity $\alpha_{s}$ depends on structure of the porous medium. The validity of the condition (8) has been widely supported [1, 8].

\section{Solution}

To solve the boundary value problem for the outer region, (1)-(3) Eq. (1) is reduced to two equations of second order:

$$
\begin{aligned}
& \Delta \psi^{e}=\eta, \\
& \Delta \eta=0,
\end{aligned}
$$


65 where $\eta=-\omega^{e}$. By using the Rayleigh-Green biharmonic boundary formula (see 9]) and Green's second identity, we obtain the equivalent pair of coupled integral equations [10],

$$
\begin{aligned}
\chi(x, y) \psi^{e}(x, y)= & \int_{\Gamma^{e}}\left(\psi^{e}(s) G_{1}^{\prime}(x, y, s)-\psi^{e^{\prime}}(s) G_{1}(x, y, s)+\right. \\
& \left.+\eta(s) G_{2}^{\prime}(x, y, s)-\eta^{\prime}(s) G_{2}(x, y, s)\right) d s \\
\chi(x, y) \eta(x, y)= & \int_{\Gamma^{e}}\left(\eta(s) G_{1}^{\prime}(x, y, s)-\eta^{\prime}(s) G_{1}(x, y, s)\right) d s
\end{aligned}
$$

where $\chi(x, y)=2 \pi$ for the interior points $(x, y) \in \Omega^{e}, \chi(x, y)=\beta$ for the boundary points $(x, y) \in \Gamma^{e}(\beta$ is the interior angle at a point on the boundary $\left.\Gamma^{e}\right)$, a prime denotes differentiation with respect to the outward normal to the boundary $\Gamma^{e}, s$ is the boundary arc. The Greens functions are written as

$G_{1}=\ln \rho, \quad G_{2}=\frac{\rho^{2}}{4}(\ln \rho-1), \quad \rho(x, y, s)=\sqrt{\left(x_{1}(s)-x\right)^{2}+\left(y_{1}(s)-y\right)^{2}}$, where $\left(x_{1}, y_{1}\right)$ is the coordinate of the boundary point with arc abscissa $s$.

The boundary $\Gamma^{e}=\bigcup_{j=1}^{n} \Gamma_{j}^{e}$ is presented as a number of linear elements $\Gamma_{j}^{e}$ 70 (linear segments). The functions $\psi^{e}(s), \psi^{e^{\prime}}(s), \eta(s), \eta^{\prime}(s)$ are approximated by piece-wise constant functions with values $\psi_{j}^{e}, \psi_{j}^{e^{\prime}}, \eta_{j}, \eta_{j}^{\prime}$ on the single element $\Gamma_{j}^{e}$. Eq. (9) can be rewritten in the discrete form

$$
\begin{aligned}
\chi(x, y) \psi^{e}(x, y)= & \sum_{j=1}^{n}\left\{\psi_{j}^{e} \int_{\Gamma_{j}^{e}} G_{1}^{\prime}(x, y, s) d s-\psi_{j}^{e^{\prime}} \int_{\Gamma_{j}^{e}} G_{1}(x, y, s) d s+\right. \\
& \left.+\eta_{j} \int_{\Gamma_{j}^{e}} G_{2}^{\prime}(x, y, s) d s-\eta_{j}^{\prime} \int_{\Gamma_{j}^{e}} G_{2}(x, y, s) d s\right\} \\
\chi(x, y) \eta(x, y)= & \sum_{j=1}^{n}\left\{\eta_{j} \int_{\Gamma_{j}^{e}} G_{1}^{\prime}(x, y, s) d s-\eta_{j}^{\prime} \int_{\Gamma_{j}^{e}} G_{1}(x, y, s) d s\right\} .
\end{aligned}
$$

Applying the expressions (10) at the centers of the elements $\Gamma_{i}^{e},\left(x_{c i}, y_{c i}\right)$, 
gives

$$
\begin{aligned}
& \sum_{j=1}^{n}\left\{\psi_{j}^{e} A_{i j}+\psi_{j}^{e \prime} B_{i j}+\eta_{j} C_{i j}+\eta_{j}^{\prime} D_{i j}\right\}=0 \\
& \sum_{j=1}^{n}\left\{\eta_{j} A_{i j}+\eta_{j}^{\prime} B_{i j}\right\}=0
\end{aligned}
$$

where

$$
\begin{array}{rlrl}
A_{i j} & =\int_{\Gamma_{j}^{e}} G_{1}^{\prime}\left(x_{c i}, y_{c i}, s\right) d s-\beta_{i} \delta_{i j}, & B_{i j} & =-\int_{\Gamma_{j}^{e}} G_{1}\left(x_{c i}, y_{c i}, s\right) d s, \\
C_{i j}=\int_{\Gamma_{j}^{e}} G_{2}^{\prime}\left(x_{c i}, y_{c i}, s\right) d s, & D_{i j}=-\int_{\Gamma_{j}^{e}} G_{2}\left(x_{c i}, y_{c i}, s\right) d s,
\end{array}
$$

75 where $\beta_{i}=\beta\left(x_{c i}, y_{c i}\right)$ and $\delta_{i j}$ are the Kronecker symbols.

The stream function $\psi^{i}(x, y)$, that is described by Eq. (4), can be written in the form

$$
\chi(x, y) \psi^{i}(x, y)=\int_{\Gamma^{i}}\left(\psi^{i}(s) G_{1}^{\prime}(x, y, s)-\psi^{i^{\prime}}(s) G_{1}(x, y, s)\right) d s .
$$

Dividing the boundary into linear elements such that $\Gamma^{i}=\bigcup_{j=1}^{m} \Gamma_{j}^{i}$ we obtain

$$
\chi(x, y) \psi^{i}(x, y)=\sum_{j=1}^{m}\left\{\psi_{j}^{i} \int_{\Gamma_{j}^{i}} G_{1}^{\prime}(x, y, s) d s-\psi_{j}^{i^{\prime}} \int_{\Gamma_{j}^{i}} G_{1}(x, y, s) d s\right\} .
$$

Equation (12) can be written in the form

$$
\begin{gathered}
\sum_{j=1}^{m}\left\{\psi_{j}^{i} E_{k j}+\psi_{j}^{i^{\prime}} F_{k j}\right\}=0 \\
E_{k j}=\int_{\Gamma_{j}^{i}} G_{1}^{\prime}\left(x_{c k}, y_{c k}, s\right) d s-\beta_{k} \delta_{k j}, \quad F_{k j}=-\int_{\Gamma_{j}^{i}} G_{1}\left(x_{c k}, y_{c k}, s\right) d s
\end{gathered}
$$

where the points $\left(x_{c k}, y_{c k}\right)$ are the centers of the elements $\Gamma_{k}^{i}$.

The obtained Eqs. (11) and (13) are a system of linear algebraic equations

80 (SLAE). The boundary conditions (2), (3), (5) are written as

$$
\begin{aligned}
& \psi_{j}^{e}=y_{c j}, \eta_{j}=0 \text { at } A D, \\
& \psi_{j}^{e}=0, \eta_{j}=0 \text { at } A B C D,
\end{aligned}
$$


$\psi_{k}^{i}=0$, at $B O C$.

The conditions (6) and (8) on the porous cylinder surface $B C$ are expressed in

terms of the stream function and vorticity:

$$
\begin{gathered}
\frac{\partial \omega^{e}}{\partial r}=S^{2} \frac{\partial \psi^{i}}{\partial r} \\
\Delta \psi^{e}-\frac{\partial \psi^{e}}{\partial r}-\frac{\partial^{2} \psi^{e}}{\partial \theta^{2}}=\alpha_{s} S\left(\frac{\partial \psi^{e}}{\partial r}-\frac{\partial \psi^{i}}{\partial r}\right) .
\end{gathered}
$$

From (14) and (15) the following equations can be obtained:

$$
\begin{gathered}
\eta_{j}^{\prime}-S^{2} \psi_{k}^{i}=0, \quad \psi_{j}^{e}-\psi_{k}^{i}=0, \\
\eta_{j}+\left(1+\alpha_{s} S\right) \psi_{j}^{e \prime}-\frac{\psi_{j-1}^{e}-2 \psi_{j}^{e}+\psi_{j+1}^{e}}{\Delta \theta^{2}}+\alpha_{s} S \psi_{k}^{i^{\prime}}=0,
\end{gathered}
$$

where $j$ and $k$ correspond to the indices of the same linear elements of the boundaries $\Gamma^{e}$ and $\Gamma^{i}$. For the domains $\Omega^{e}$ and $\Omega^{i}$ we have $\partial / \partial n=-\partial / \partial r$ and $\partial / \partial n=\partial / \partial r$ respectively. To approximate $\partial^{2} \psi^{e} / \partial \theta^{2}$ the finite difference relation is used under the assumption that the linear elements on $B C$ have equal lengths $(\Delta \theta$ is the difference between the angle coordinates of adjacent linear elements). The same conditions were used by Dunnett \& Clement [3].

The SLAE obtained is then solved numerically to find the unknown quantities $\psi_{j}^{e}, \psi_{j}^{e^{\prime}}, \eta_{j}, \eta_{j}^{\prime}, j=\overline{1, n}, \psi_{k}^{i}, \psi_{k}^{i^{\prime}}, k=\overline{1, m}$. The functions $\psi^{e}(x, y)$, $\omega^{e}(x, y), \psi^{i}(x, y)$ at the general point $(x, y)$ can then be calculated from the formulas (10), (12). Taking into account $u=\partial \psi / \partial y, v=-\partial \psi / \partial x$ the formulas for velocity components are written:

$$
\begin{aligned}
\chi(x, y) u^{e}(x, y)= & \sum_{j=1}^{n}\left\{\psi_{j}^{e} \int_{\Gamma_{j}^{e}} \frac{\partial G_{1}^{\prime}(x, y, s)}{\partial y} d s-\psi_{j}^{e \prime} \int_{\Gamma_{j}^{e}} \frac{\partial G_{1}(x, y, s)}{\partial y} d s+\right. \\
& \left.+\eta_{j} \int_{\Gamma_{j}^{e}} \frac{\partial G_{2}^{\prime}(x, y, s)}{\partial y} d s-\eta_{j}^{\prime} \int_{\Gamma_{j}^{e}} \frac{\partial G_{2}(x, y, s)}{\partial y} d s\right\}, \\
\chi(x, y) v^{e}(x, y)= & -\sum_{j=1}^{n}\left\{\psi_{j}^{e} \int_{\Gamma_{j}^{e}} \frac{\partial G_{1}^{\prime}(x, y, s)}{\partial x} d s-\psi_{j}^{e \prime} \int_{\Gamma_{j}^{e}} \frac{\partial G_{1}(x, y, s)}{\partial x} d s+\right.
\end{aligned}
$$




$$
\begin{gathered}
\left.+\eta_{j} \int_{\Gamma_{j}^{e}} \frac{\partial G_{2}^{\prime}(x, y, s)}{\partial x} d s-\eta_{j}^{\prime} \int_{\Gamma_{j}^{e}} \frac{\partial G_{2}(x, y, s)}{\partial x} d s\right\}, \\
\chi(x, y) u^{i}(x, y)=\sum_{j=1}^{m}\left\{\psi_{j}^{i} \int_{\Gamma_{j}^{i}} \frac{\partial G_{1}^{\prime}(x, y, s)}{\partial y} d s-\psi_{j}^{i^{\prime}} \int_{\Gamma_{j}^{i}} \frac{\partial G_{1}(x, y, s)}{\partial y} d s\right\}, \\
\chi(x, y) v^{i}(x, y)=-\sum_{j=1}^{m}\left\{\psi_{j}^{i} \int_{\Gamma_{j}^{i}} \frac{\partial G_{1}^{\prime}(x, y, s)}{\partial x} d s-\psi_{j}^{i^{\prime}} \int_{\Gamma_{j}^{i}} \frac{\partial G_{1}(x, y, s)}{\partial x} d s\right\} .
\end{gathered}
$$

In order to solve equations (10)-(13), (16) it is necessary to calculate the various integrals in these equations.

In order to achieve this we introduce the following notation:

$$
f_{1}=G_{1}, \quad f_{2}=G_{2} .
$$

100 Let $\varphi$ be the angle of the tangent slope to the panel $\Gamma_{j}$. The angle $\varphi$ is constant for each panel. Then

$$
\frac{d x_{1}}{d n}=\sin \varphi, \quad \frac{d y_{1}}{d n}=-\cos \varphi .
$$

The other functions in the integrals can be obtained by differentiating the functions $G_{1}$ and $G_{2}$ taking into account (17):

$$
\begin{gathered}
f_{3}=G_{1}^{\prime}=\frac{\left(x_{1}-x\right) \sin \varphi-\left(y_{1}-y\right) \cos \varphi}{\rho^{2}} \\
f_{4}=G_{2}^{\prime}=\frac{1}{4}\left[\left(x_{1}-x\right) \sin \varphi-\left(y_{1}-y\right) \cos \varphi\right](2 \ln \rho-1), \\
f_{5}=\frac{d G_{1}}{d x}=-\frac{x_{1}-x}{\rho^{2}}, \quad f_{6}=\frac{d G_{1}}{d y}=-\frac{y_{1}-y}{\rho^{2}}, \\
f_{7}=\frac{d G_{1}^{\prime}}{d x}=\frac{\left[\left(x_{1}-x\right)^{2}-\left(y_{1}-y\right)^{2}\right] \sin \varphi-2\left(x_{1}-x\right)\left(y_{1}-y\right) \cos \varphi}{\rho^{4}} \\
f_{8}=\frac{d G_{1}^{\prime}}{d y}=\frac{\left[\left(x_{1}-x\right)^{2}-\left(y_{1}-y\right)^{2}\right] \cos \varphi+2\left(x_{1}-x\right)\left(y_{1}-y\right) \sin \varphi}{\rho^{4}} \\
f_{9}=\frac{d G_{2}}{d x}=-\frac{1}{4}\left(x_{1}-x\right)(2 \ln \rho-1), \quad f_{10}=\frac{d G_{2}}{d y}=-\frac{1}{4}\left(y_{1}-y\right)(2 \ln \rho-1), \\
f_{11}=\frac{d G_{2}^{\prime}}{d x}=\frac{1}{4}\left[-\left(2 \ln \rho-1+\frac{2\left(x_{1}-x\right)^{2}}{\rho^{2}}\right) \sin \varphi+\frac{2\left(x_{1}-x\right)\left(y_{1}-y\right)}{\rho^{2}} \cos \varphi\right]
\end{gathered}
$$




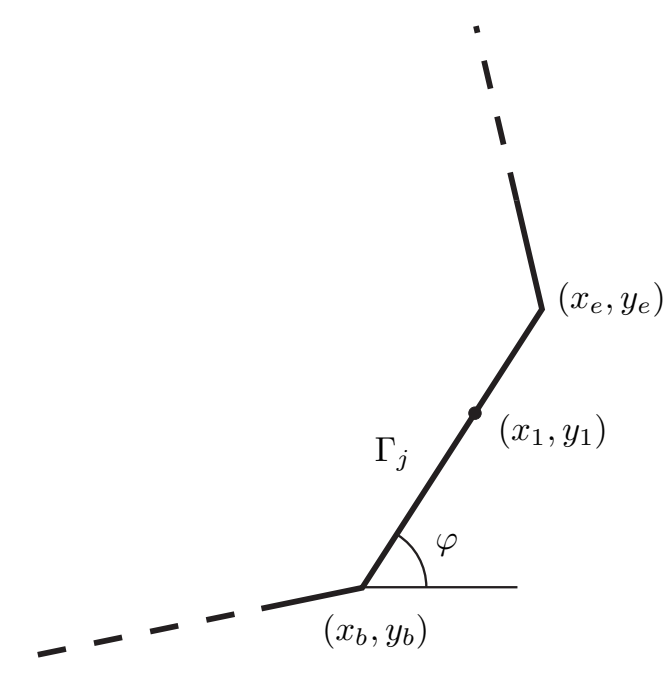

Figure 2: Single panel scheme

$$
f_{12}=\frac{d G_{2}^{\prime}}{d y}=\frac{1}{4}\left[\left(2 \ln \rho-1+\frac{2\left(x_{1}-x\right)^{2}}{\rho^{2}}\right) \cos \varphi-\frac{2\left(x_{1}-x\right)\left(y_{1}-y\right)}{\rho^{2}} \sin \varphi\right] .
$$

Calculating the integrals from the 12 functions above can be achieved numerically but it is time consuming when solving the SLAE and determining the fluid flow velocity at the current point of the calculation domain. Because of this the analytical determination of the integral would be preferable. The integrals involving the first four functions $f_{i}, i=\overline{1,4}$ have been determined analytically previously, see [10]. In this work we will give analytical expressions for all the other integrals needed using the functions above.

Denoting the coordinates of the ends of the panels $\Gamma_{j},\left(x_{b}, y_{b}\right)$ and $\left(x_{e}, y_{e}\right)$ respectively (fig. 2). The coordinates of the current point with arc abscissa $s$ can be written as

$$
x_{1}(s)=x_{b}+\left(s-s_{b}\right) \cos \varphi, \quad y_{1}(s)=y_{b}+\left(s-s_{b}\right) \sin \varphi,
$$

where $s_{b}$ is the arc abscissa of the point $\left(x_{b}, y_{b}\right)$.

Introducing the complex coordinates

$$
z=x+i y, \quad z_{1}=x_{1}+i y_{1}, \quad z_{b}=x_{b}+i y_{b}, \quad z_{e}=x_{e}+i y_{e}
$$


and variables

$$
\zeta=z_{1}-z, \quad \zeta_{b}=z_{b}-z, \quad \zeta_{e}=z_{e}-z
$$

Note that $\rho=\left|z_{1}-z\right|=|\zeta|$. Hence we can write

$$
G_{1}=\operatorname{Re}\{\ln \zeta\}
$$

Taking into account that in the panel points

$$
d \zeta=d z_{1}=e^{i \varphi} d s
$$

then

$$
\begin{aligned}
F_{1}=\int_{\Gamma_{j}} G_{1} d s & =\operatorname{Re}\left\{\int_{\Gamma_{j}} \ln \zeta d s\right\}=\operatorname{Re}\left\{e^{-i \varphi} \int_{\zeta_{b}}^{\zeta_{e}} \ln \zeta d \zeta\right\}= \\
& =\operatorname{Re}\left\{e^{\left.-i \varphi[\zeta(\ln \zeta-1)]_{\zeta_{b}}^{\zeta_{e}}\right\} .}\right.
\end{aligned}
$$

Introducing the notation

$$
[f(\zeta)]_{\zeta_{b}}^{\zeta_{e}}=f\left(\zeta_{e}\right)-f\left(\zeta_{b}\right)
$$

The derivative with respect to the normal can be written as

$$
\frac{d}{d n}=-i e^{i \varphi} \frac{d}{d \zeta}
$$

where $d \zeta=d z_{1}=e^{i(\varphi-\pi / 2)} d n$. Then from (20):

$$
G_{1}^{\prime}=\operatorname{Re}\left\{\frac{d(\ln \zeta)}{d n}\right\}=\operatorname{Re}\left\{-i e^{i \varphi} \frac{d(\ln \zeta)}{d \zeta}\right\}=\operatorname{Im}\left\{\frac{e^{i \varphi}}{\zeta}\right\}
$$

Integrating the function $G_{1}^{\prime}$ gives:

$$
F_{3}=\int_{\Gamma_{j}} G_{1}^{\prime} d s=\operatorname{Im}\left\{e^{-i \varphi} \int_{\zeta_{b}}^{\zeta_{e}} \frac{e^{i \varphi}}{\zeta} d \zeta\right\}=\operatorname{Im}\left\{[\ln \zeta]_{\zeta_{b}}^{\zeta_{e}}\right\} .
$$

Taking into account $\rho^{2}=\zeta \bar{\zeta}$ the function $G_{2}$ can be written as

$$
G_{2}=\frac{1}{4} \operatorname{Re}\{\zeta \bar{\zeta}(\ln \zeta-1)\}
$$


Thus

$$
\int_{\Gamma_{j}} G_{2} d s=\frac{1}{4} \operatorname{Re}\left\{e^{-i \varphi} \int_{\zeta_{b}}^{\zeta_{e}} \zeta \bar{\zeta}(\ln \zeta-1) d \zeta\right\}
$$

In order to calculate this integral integration by parts is used.

$$
\int u d v=u v-\int v d u
$$

Taking $u=\bar{\zeta}, d v=\zeta(\ln \zeta-1) d \zeta$ and taking into account the formulas (21) and the fact $d \bar{\zeta}=e^{-i \varphi} d s$ we have

$$
\frac{d \bar{\zeta}}{d \zeta}=e^{-2 i \varphi}
$$

The quantities $d u$ and $v$ can be written in the form: $d u=e^{-2 i \varphi} d \zeta, v=\zeta^{2}(2 \ln \zeta-3) / 4$.

Inserting these expressions into the integral we obtain

$$
F_{2}=\int_{\Gamma_{j}} G_{2} d s=\frac{1}{16} \operatorname{Re}\left\{e^{-i \varphi}\left[\zeta^{2} \bar{\zeta}(2 \ln \zeta-3)-\frac{\zeta^{3}}{9}(6 \ln \zeta-11) e^{-2 i \varphi}\right]_{\zeta_{b}}^{\zeta_{e}}\right\}
$$

To calculate $G_{2}^{\prime}$, from (23) it follows that $d \bar{\zeta}=i e^{-i \varphi} d n$. Hence

$$
\frac{d \bar{\zeta}}{d \zeta}=-e^{-2 i \varphi} .
$$

Differentiating (25) and taking into account (23) and (27) we have

$$
G_{2}^{\prime}=\frac{1}{4} \operatorname{Im}\left\{e^{i \varphi} \bar{\zeta} \ln \zeta-e^{-i \varphi} \zeta(\ln \zeta-1)\right\}
$$

Then

$$
\int_{\Gamma_{j}} G_{2}^{\prime} d s=\frac{1}{4} \operatorname{Im}\left\{\int_{\zeta_{b}}^{\zeta_{e}} \bar{\zeta} \ln \zeta d \zeta-e^{-2 i \varphi} \int_{\zeta_{b}}^{\zeta_{e}} \zeta(\ln \zeta-1) d \zeta\right\} .
$$

Computing the integrals we obtain

$$
F_{4}=\int_{\Gamma_{j}} G_{2}^{\prime} d s=\frac{1}{4} \operatorname{Im}\left\{\left[\zeta \bar{\zeta}(\ln \zeta-1)-\frac{\zeta^{2}}{2}(2 \ln \zeta-3) e^{-2 i \varphi}\right]_{\zeta_{b}}^{\zeta_{e}}\right\} .
$$

To integrate the other 8 integrals we differentiate (22), (24), (26), (28) taking into account the formulas obtained from (18) and (19)

$$
\frac{d \zeta}{d x}=-1, \quad \frac{d \bar{\zeta}}{d x}=-1, \quad \frac{d \zeta}{d y}=-i, \quad \frac{d \bar{\zeta}}{d y}=i
$$




$$
\begin{aligned}
& \int_{\Gamma_{j}} \frac{d G_{1}}{d x} d s=\frac{d F_{1}}{d x}=-\operatorname{Re}\left\{e^{-i \varphi}[\ln \zeta]_{\zeta_{b}}^{\zeta_{e}}\right\}, \\
& \int_{\Gamma_{j}} \frac{d G_{1}}{d y} d s=\frac{d F_{1}}{d y}=\operatorname{Im}\left\{e^{-i \varphi}[\ln \zeta]_{\zeta_{b}}^{\zeta_{e}}\right\} \\
& \int_{\Gamma_{j}} \frac{d G_{1}^{\prime}}{d x} d s=\frac{d F_{3}}{d x}=-\operatorname{Im}\left\{\left[\frac{1}{\zeta}\right]_{\zeta_{b}}^{\zeta_{e}}\right\} \\
& \int_{\Gamma_{j}} \frac{d G_{1}^{\prime}}{d y} d s=\frac{d F_{3}}{d y}=-\operatorname{Re}\left\{\left[\frac{1}{\zeta}\right]_{\zeta_{b}}^{\zeta_{e}}\right\} \\
& \int_{\Gamma_{j}} \frac{d G_{2}}{d x} d s=\frac{d F_{2}}{d x}= \\
& =\frac{1}{16} \operatorname{Re}\left\{e^{-i \varphi}\left[-4 \zeta \bar{\zeta}(\ln \zeta-1)+\zeta^{2}(2 \ln \zeta-3)\left(e^{-2 i \varphi}-1\right)\right]_{\zeta_{b}}^{\zeta_{e}}\right\}, \\
& \int_{\Gamma_{j}} \frac{d G_{2}}{d y} d s=\frac{d F_{2}}{d y}= \\
& =-\frac{1}{16} \operatorname{Im}\left\{e^{-i \varphi}\left[-4 \zeta \bar{\zeta}(\ln \zeta-1)+\zeta^{2}(2 \ln \zeta-3)\left(e^{-2 i \varphi}+1\right)\right]_{\zeta_{b}}^{\zeta_{e}}\right\}, \\
& \int_{\Gamma_{j}} \frac{d G_{2}^{\prime}}{d x} d s=\frac{d F_{4}}{d x}=\frac{1}{4} \operatorname{Im}\left\{\left[-\bar{\zeta} \ln \zeta+\zeta(\ln \zeta-1)\left(2 e^{-2 i \varphi}-1\right)\right]_{\zeta_{b}}^{\zeta_{e}}\right\}, \\
& \int_{\Gamma_{j}} \frac{d G_{2}^{\prime}}{d y} d s=\frac{d F_{4}}{d y}=\frac{1}{4} \operatorname{Re}\left\{\left[-\bar{\zeta} \ln \zeta+\zeta(\ln \zeta-1)\left(2 e^{-2 i \varphi}+1\right)\right]_{\zeta_{b}}^{\zeta_{e}}\right\} .
\end{aligned}
$$

The formulas obtained can be used for $z \notin \Gamma_{j}$ as well for the case $z \in \Gamma_{j}$ when some of integrals are singular and they can not to be integrated numerically. For the case $z \notin \Gamma_{j}$ the increment of the argument $\zeta$ at the movement of $z_{1}$ along the panel $\Gamma_{j}$ must be calculated keeping the selected branch of the function $\arg \zeta$. The increment of the argument $\zeta$ is accepted equal to zero for $z \in \Gamma_{j}$. It corresponds to the calculations in terms of principal value of singular integrals. 


\section{Analytical solution for Kuwabara cell}

The problem of fluid flow past a porous cylinder in the periodic cell of the Kuwabara model can be solved analytically [1]. We will seek a solution of the form

$$
\psi_{a}^{e}(r, \theta)=\left(A \frac{1}{r}+B r+C r \ln r+D r^{3}\right) \sin \theta, \quad \psi_{a}^{i}(r, \theta)=G r \sin \theta .
$$

Taking into account the boundary conditions (22), (7), (14), (15) and the fact that $\omega^{e}(x, y)=-\Delta \psi^{e}$ the coefficients $A, B, C, D, G$ can be obtained as:

$$
\begin{gathered}
A=S^{2} k_{1}^{-1}\left(2-3 \gamma+\alpha_{s} S(\gamma-2)\right), \\
B=-2 k_{1}^{-1}\left(4(1+\gamma)\left(1+\alpha_{s} S\right)+S^{2}(1-\gamma)\left(1-\alpha_{s} S\right)\right), \\
C=-8 k^{-1}\left(1+\alpha_{s} S\right), \quad D=2 \alpha_{s} k^{-1}\left(1+\alpha_{s} S\right), \\
G=-8 S^{-2} k^{-1}\left(1+\alpha_{s}\right)\left(1+\alpha_{s} S\right),
\end{gathered}
$$

where $\gamma=h^{-2}$,

$k=-2+8 \gamma-6 \gamma^{2}+\alpha_{s} S\left(6-8 \gamma+2 \gamma^{2}\right)-16 S^{-2}(1+\gamma)\left(1+\alpha_{s} S\right)+4\left(1+\alpha_{s} S\right) \ln \gamma$,

$k_{1}=-8(1+\gamma)\left(1+\alpha_{s} S\right)+4 \gamma S^{2}\left(1-\alpha_{s} S\right)+3 S^{2}\left(\gamma^{2}-\alpha_{s} S\right)+\gamma^{2} \alpha_{s} S^{3}+2 S^{2}\left(1+\alpha_{s} S\right) \ln \gamma$.

The velocity components and vorticity can be written in the form

$$
\begin{aligned}
u_{a r}^{e}(r, \theta) & =\frac{1}{r} \frac{\partial \psi_{a}^{e}}{\partial \theta}=\left(\frac{A}{r^{2}}+B+C \ln r+D r^{2}\right) \cos \theta, \\
u_{a r}^{i}(r, \theta) & =\frac{1}{r} \frac{\partial \psi_{a}^{i}}{\partial \theta}=G \cos \theta \\
u_{a \theta}^{e}(r, \theta) & =-\frac{\partial \psi_{a}^{e}}{\partial r}=-\left(-\frac{A}{r^{2}}+B+C(\ln r+1)+3 D r^{2}\right) \sin \theta, \\
u_{a \theta}^{i}(r, \theta) & =-\frac{\partial \psi_{a}^{i}}{\partial r}=-G \sin \theta, \\
\omega_{a}^{e}(r, \theta) & =\frac{1}{r} \frac{\partial}{\partial r}\left(r \frac{\partial \psi_{a}^{e}}{\partial r}\right)+\frac{1}{r^{2}} \frac{\partial^{2} \psi_{a}^{e}}{\partial r^{2}}=-2\left(\frac{C}{r}+4 D r\right) \sin \theta, \\
\omega_{a}^{i}(r, \theta) & \equiv 0 .
\end{aligned}
$$




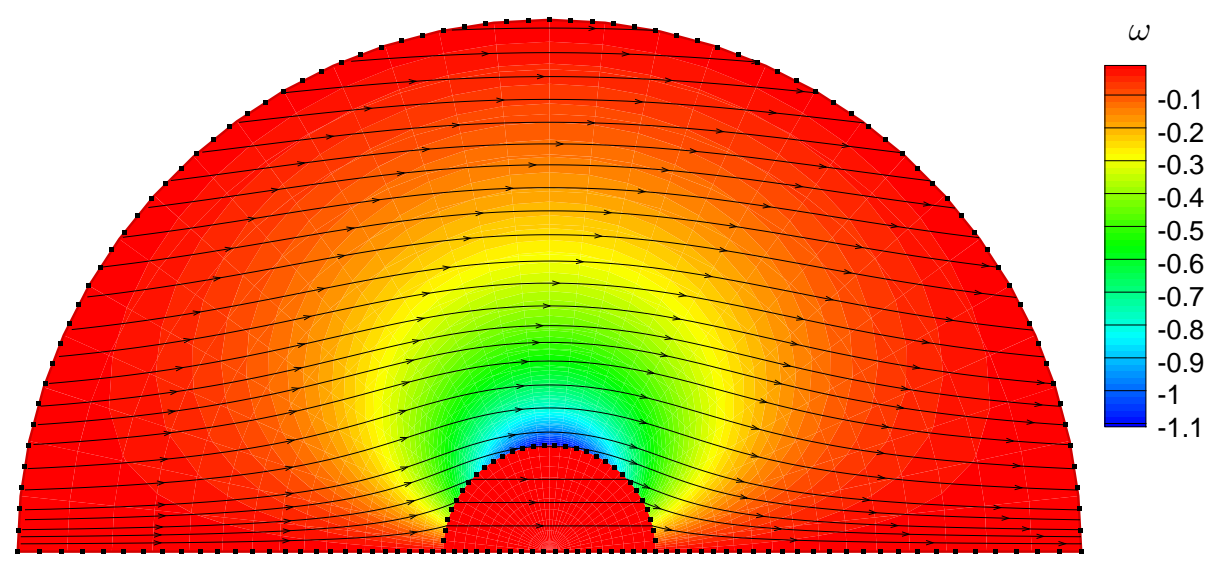

Figure 3: Streamlines and vorticity distributions

\section{Numerical results}

To test the developed numerical model the values $h=5, \alpha_{s}=1, S=2$ are used. The number of linear elements on the boundaries $\Gamma^{e}$ and $\Gamma^{i}$ is taken to be $n=161$ and $m=50$ respectively.

The distributions of streamlines and vorticity obtained are given in fig. 3 The points on the boundaries show the ends of the linear elements of $\Gamma^{e}$ and $\Gamma^{i}$. Note that the distributions obtained from analytical formulas are not shown because they practically coincide with the presented numerical distributions.

To estimate the accuracy of the method the absolute $\left(E_{\psi}(x, y), E_{u}(x, y)\right.$, $\left.E_{v}(x, y), E_{\omega}(x, y)\right)$ and relative $\left(\varepsilon_{\psi}, \varepsilon_{u}, \varepsilon_{v}, \varepsilon_{\omega}\right)$ errors were calculated for the functions $\psi(x, y), u(x, y), v(x, y), \omega(x, y)$ :

$$
\begin{gathered}
E_{\psi}(x, y)=\left|\psi(x, y)-\psi_{a}(x, y)\right|, \quad E_{u}(x, y)=\left|u(x, y)-u_{a}(x, y)\right|, \\
E_{v}(x, y)=\left|v(x, y)-v_{a}(x, y)\right|, \quad E_{\omega}(x, y)=\left|\omega(x, y)-\omega_{a}(x, y)\right|, \\
\varepsilon_{\psi}=\frac{\max E_{\psi}(x, y)}{\max \left|\psi_{a}(x, y)\right|}, \quad \varepsilon_{u}=\frac{\max E_{u}(x, y)}{\max \sqrt{u_{a}^{2}(x, y)+v_{a}^{2}(x, y)}}, \\
\varepsilon_{v}=\frac{\max E_{v}(x, y)}{\max \sqrt{u_{a}^{2}(x, y)+v_{a}^{2}(x, y)}}, \quad \varepsilon_{\omega}=\frac{\max E_{\omega}(x, y)}{\max \left|\omega_{a}(x, y)\right|} .
\end{gathered}
$$



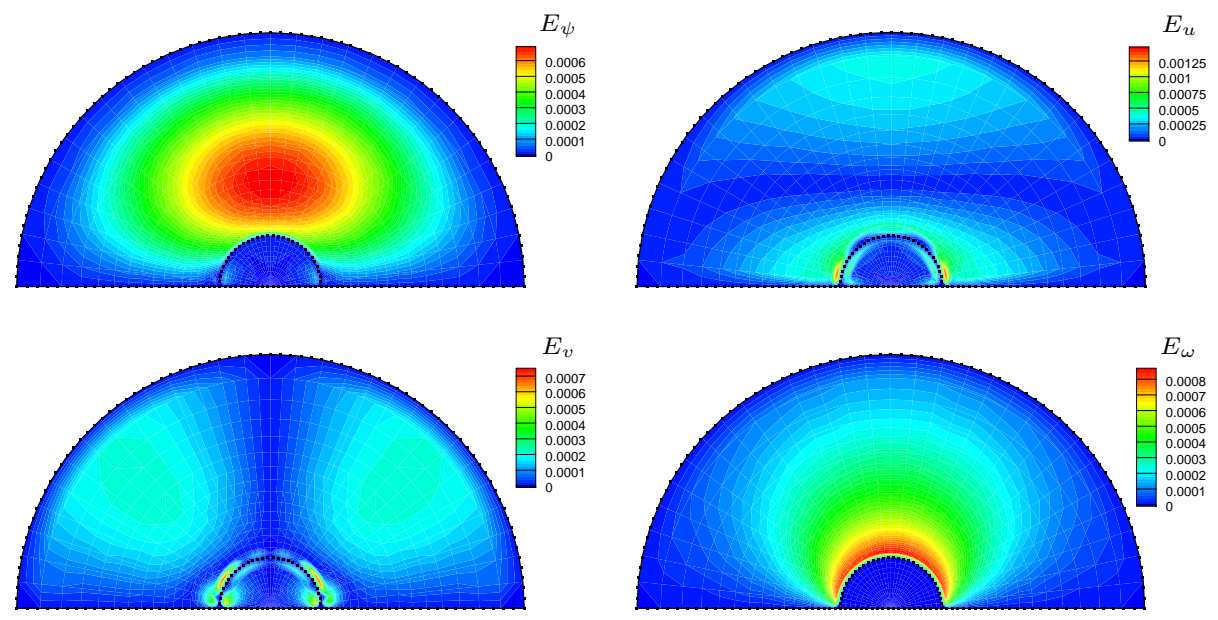

Figure 4: The distributions $E_{\psi}(x, y), E_{u}(x, y), E_{v}(x, y), E_{\omega}(x, y)$
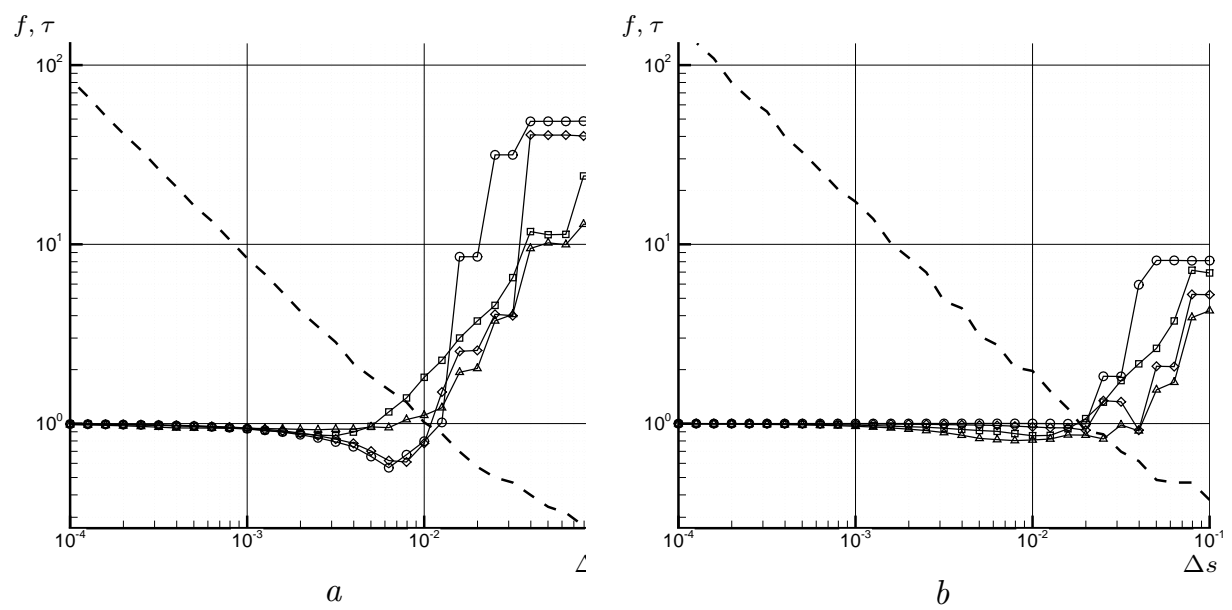

Figure 5: The errors ratio as a function of $\Delta s: \square-f_{\psi}, \Delta-f_{\omega}, \diamond-f_{u}, \circ-f_{v}$

The distributions $E_{\psi}(x, y), E_{u}(x, y), E_{v}(x, y), E_{\omega}(x, y)$ are shown in fig. [ The relative errors are $\varepsilon_{\psi}=0.00014, \varepsilon_{u}=0.00112, \varepsilon_{v}=0.00057, \varepsilon_{\omega}=0.00086$.

The observed small values of absolute and relative errors confirm the high accuracy of the developed method.

The results of the comparison of using analytical (method 1) and numerical (method 2) integration are given in fig. 5 . On the figure the $x$-axis is the 
integration step $\Delta s$ within one boundary element. Ordinates are the errors ratio. The ratio's of the errors $E$ obtained by method 1, index $a$, and method 2, index $n$, are shown for $\psi, \omega, u, v$ :

$$
\begin{aligned}
f_{\psi} & =\frac{\max E_{\psi}^{n}(x, y)}{\max E_{\psi}^{a}(x, y)}, \\
f_{u} & =\frac{\max E_{u}^{n}(x, y)}{\max E_{u}^{a}(x, y)},
\end{aligned}
$$

The ratio of computing times, $\tau$, for calculating the functions $\psi(x, y), u(x, y)$, $v(x, y), \omega(x, y)$ using numerical and analytical integration is shown by the dashed line in the figure.

Fig. 5. $a$ and fig. 5, $b$ correspond to the fine and coarse mesh. In the first case the length of the boundary elements on the cylinder surface $\sim 0.1$ and on the outer boundary 0.2 (the number of boundary elements for the outer domain 161, for the cylinder - 50). For the coarse mesh the length of the boundary elements on the cylinder surface $\sim 0.2$ and on the outer boundary 0.4 (the number of boundary elements for the outer domain - 80, for the cylinder -24).

It is seen that there is advantage in computing time using method 1 compared to method 2 beginning from $\Delta s>10^{-2}=0.1 / 10$ and $\Delta s>2 \cdot 10^{-2}=0.2 / 10$ for fine and coarse mesh respectively. Also, as can be seen in the figure, the accuracy of the two approaches becomes the same for $\Delta s \sim 10^{-3}$. For these values of $\Delta s$ the computing time using method 2 is $>10$ times more than for the method 1 .

The conclusion can be made that using analytical integration for determining the expressions for stream function, vorticity and velocity components in the BEM method for Stokes flow problem considerably reduces the computing time required to obtain a solution of the required accuracy.

To further test the accuracy and adaptability of the developed method two additional problems have been considered. Firstly, the problem of fluid flow through the porous cylinder in a rectangular domain, as shown in fig. 6 is studied. This corresponds to the periodic cell of a system of parallel porous cylinders such that their axes are perpendicular to the airflow. The packing 


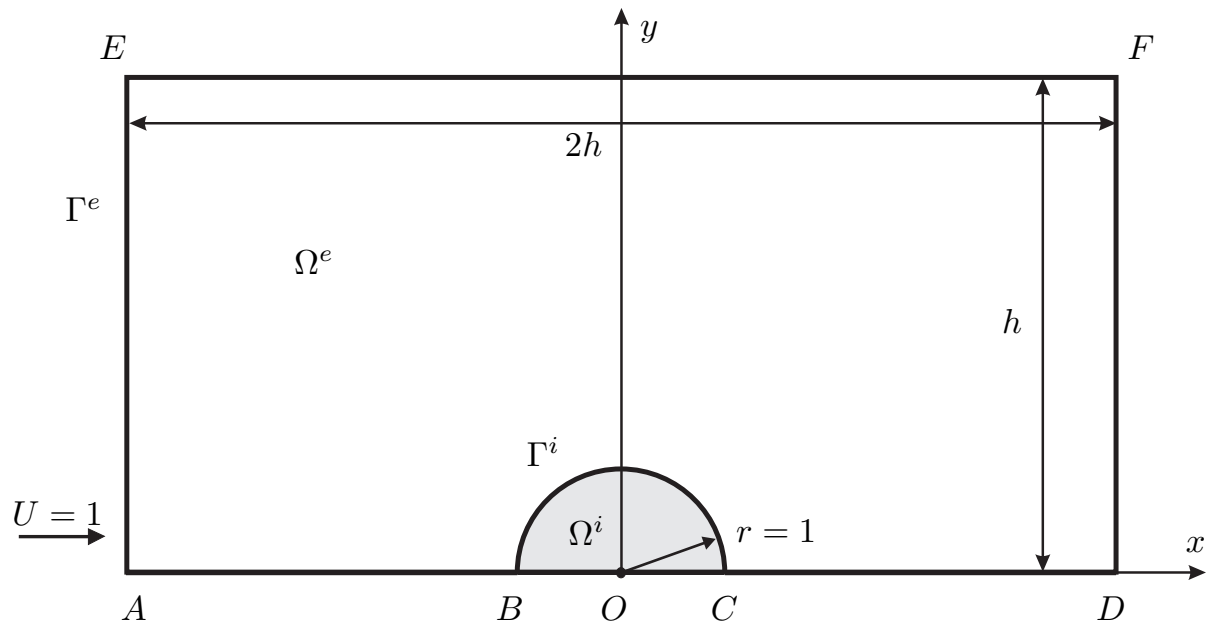

Figure 6: Fluid flow domain

fraction $\alpha$ is given by $\alpha=\pi / 4 h^{2}$. Additionally to the boundary conditions (3), (5) -(7) the conditions applied on $A E, D F$

$$
\psi^{e}=y, \quad \omega^{e}=0,
$$

and on $E F$

$$
\psi^{e}=h, \quad \omega^{e}=0 .
$$

The boundary value problem (19), (3)-(7), (29), (30) was solved using the approach developed in this paper for $h=4.43, \alpha_{s}=1, S=2$. The value $h=4.43$ is taken in order to ensure that the porosity $\varepsilon$ takes the same value, 0.96, as in the earlier results for the Kuwabara problem. The number of linear elements on the boundaries $\Gamma^{e}$ and $\Gamma^{i}$ is taken to be $n=144$ and $m=50$ respectively. It is seen from fig. 7 that the distributions of streamlines and vorticity obtained agree with the distributions in fig. 3, The relative fluid flow rate through the porous cylinder, $Q=\psi(0,1)$, is shown as a function of the parameter, $S$, for two values of $\varepsilon$ in fig. 8 . Also shown in the figure are results using the circular Kuwabara cell, fig. 1. It is seen that the quantity $Q$ decreases with increasing values of the parameter $S$ due to the decreasing permeability. 


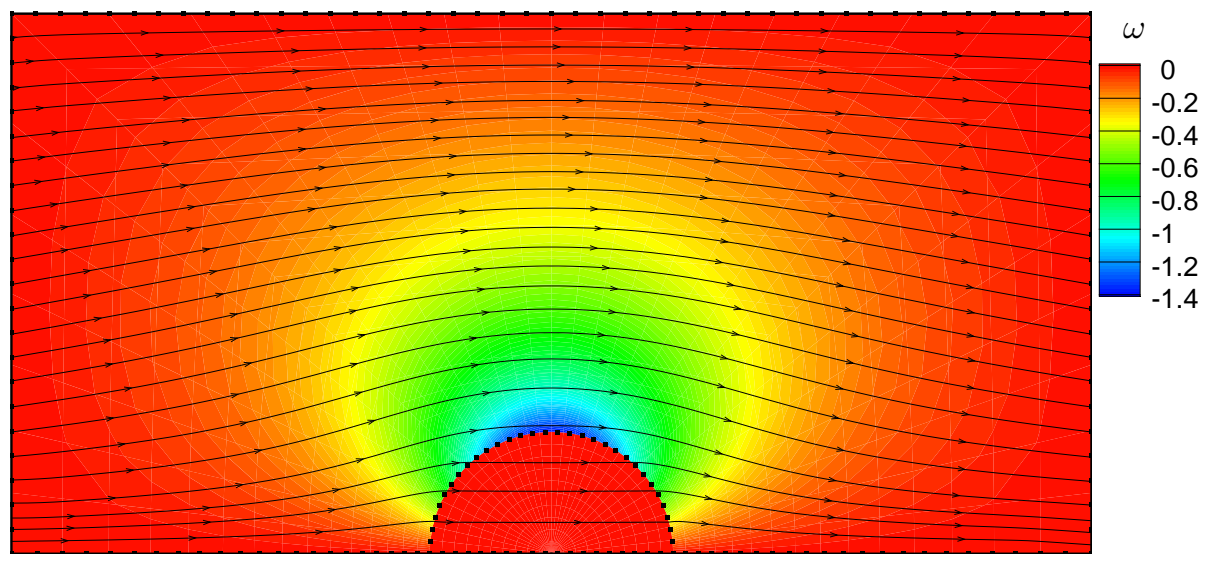

Figure 7: Streamlines and vorticity distributions

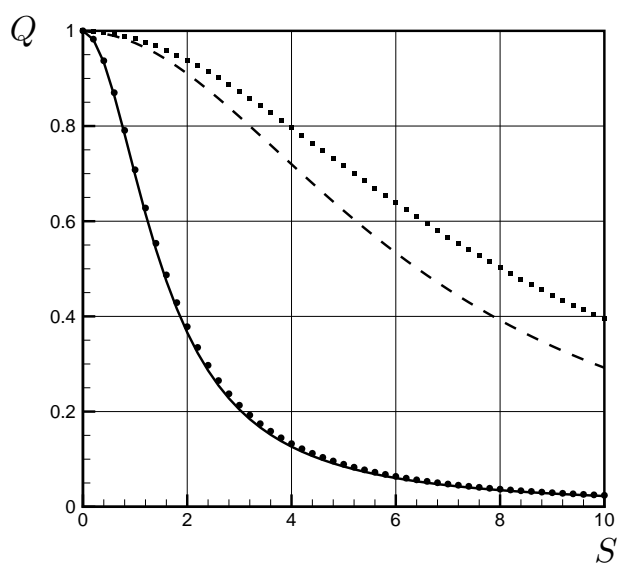

Figure 8: The relative fluid flow through porous cylinder $Q(S) . \varepsilon=0.96$ : solid line for Kuwabara cell, circle symbols for rectangle cell. $\varepsilon=0.6$ : dashed line for Kuwabara cell, square symbols for rectangle cell.

For the value $\varepsilon=0.96$ there is no significant difference in the results obtained using the rectangular and circular cells. However, $\varepsilon$ as decreases the difference between the results from the two models increases, as can be seen for $\varepsilon=0.6$ in the figure.

To validate the method developed for a doubly connected domain the flow be185 tween eccentric cylinders as considered by [11], 12], see fig. 9] has been modelled. 


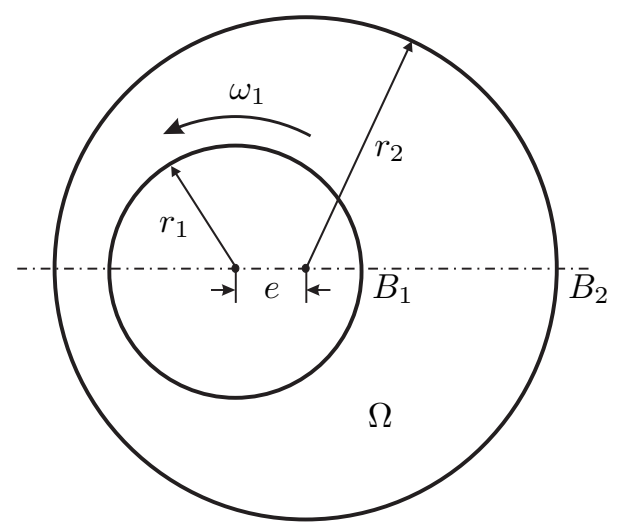

Figure 9: Fluid flow domain

The flow is described by the biharmonic equation for the stream function (11). The inner solid cylinder rotates with the constant anticlockwise angular velocity $\omega_{1}=1$ and the outer is stationary. The radii of inner and outer cylinders are $r_{1}=0.5$ and $r_{2}=2$ respectively. The eccentricity is found from the relation, $\varepsilon_{c}=e /\left(r_{2}-r_{1}\right)$ where $e$ is the distance between the centers of the cylinders.

On the inner cylinder $B_{1}$ the boundary conditions are:

$$
\begin{gathered}
\psi=\psi_{1}, \\
\frac{\partial \psi}{\partial n}=\omega_{1} r_{1} .
\end{gathered}
$$

To determine the value $\psi_{1}$ the condition for periodicity in the form taken in [11]

$$
\int_{B_{1}} \frac{\partial \psi}{\partial n} d t=0
$$

is used.

On the outer cylinder $B_{2}$ the conditions are:

$$
\begin{gathered}
\psi=0 \\
\frac{\partial \psi}{\partial n}=0 .
\end{gathered}
$$

The boundary value problem for the flow between the cylinders was solved by the BEM described above. The distributions obtained for $\psi(x, y)$ (top) and 

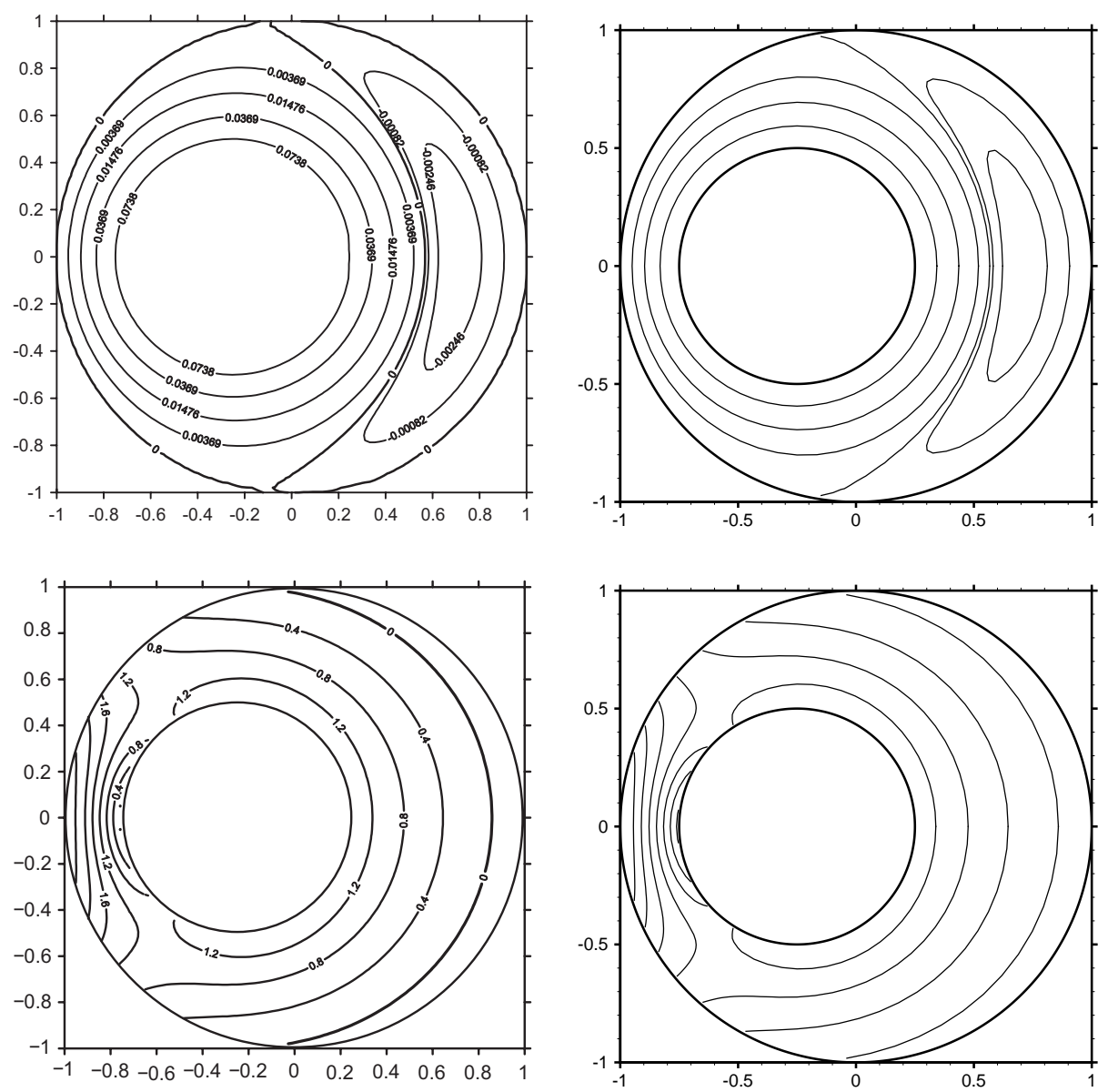

Figure 10: Comparison of distributions $\psi(x, y)$ (top) and $\omega(x, y)$ (bottom) obtained in 12] (left) and in present work (right) for $\varepsilon=0.5$

$\omega(x, y)$ (bottom) are shown in fig. 10 for $\varepsilon_{c}=0.5$. Also shown in the figure, on 195 the left hand side, are the results obtained in [12] using the boundary integral equations (BIEs) expanded in terms of Fourier series. As can be seen there is no significant difference between the distributions obtained in the earlier work and those obtained here.

Analytical and numerical results of the unknown boundary constant $\psi_{1}$ obtained in [11], 12] and in the present work are compared in Table 1. In [11] the traditional BEM was applied. The values $\psi_{1}$ obtained by the numerical 
Table 1: Comparison of analytical and numerical results

\begin{tabular}{ccccccccc}
\hline & \multicolumn{3}{c}{ Kelmanson [11] } & \multirow{2}{*}{$\begin{array}{c}\text { Analytical } \\
\text { solution }\end{array}$} & \multicolumn{3}{c}{ Present work } \\
\cline { 2 - 3 } \cline { 8 - 9 }$\varepsilon$ & $n=80$ & $n=160$ & $n=320$ & and Chen $[12]$ & $n=80$ & $n=160$ & $n=320$ \\
\hline 0.0 & 0.1066 & 0.1062 & 0.1061 & 0.1060 & 0.1056 & 0.1058 & 0.1059 \\
0.1 & 0.1052 & 0.1048 & 0.1047 & 0.1046 & 0.1042 & 0.1044 & 0.1045 \\
0.2 & 0.1011 & 0.1006 & 0.1005 & 0.1005 & 0.1001 & 0.1003 & 0.1004 \\
0.3 & 0.0944 & 0.0939 & 0.0938 & 0.0938 & 0.0935 & 0.0936 & 0.0937 \\
0.4 & 0.0854 & 0.0850 & 0.0848 & 0.0848 & 0.0845 & 0.0846 & 0.0847 \\
0.5 & 0.0748 & 0.0740 & 0.0739 & 0.0738 & 0.0736 & 0.0737 & 0.0737 \\
0.6 & 0.0622 & 0.0615 & 0.0613 & 0.0611 & 0.0610 & 0.0611 & 0.0611 \\
0.7 & 0.0484 & 0.0477 & 0.0474 & 0.0472 & 0.0471 & 0.0471 & 0.0472 \\
0.8 & 0.0347 & 0.0332 & 0.0326 & 0.0322 & 0.0322 & 0.0322 & 0.0322 \\
0.9 & 0.0191 & 0.0175 & 0.0168 & 0.0164 & -0.0055 & 0.0172 & 0.0164 \\
\hline
\end{tabular}

method of [12] are the same as analytical values and are shown in the fifth column of Table 1. It is seen that using the BEM with analytical integration, as in the present work, provides better agreement with the analytical value than the method of [11] for equal numbers $n$ of linear elements on the boundary. The negative value for $n=80$ at $\varepsilon_{c}=0.9$ is explained by the very narrow distance between cylinders that becomes approximately equal to the length of boundary linear element.

\section{Conclusion}

The viscous incompressible fluid flow in a periodic cell with a porous cylinder, the model of air flow in an aerosol filter, is considered. The Stokes flow model is adopted outside the cylinder. Inside the porous cylinder, Darcy law of drag is applied to find the filtration velocity field. The corresponding boundary value problems for the biharmonic and Laplace equations for the stream function and pressure are then formulated. The equality of pressure and normal velocity and the condition for the tangential velocity proposed by Beavers and Joseph [7] are 
taken as the boundary condition on the porous surface. The boundary elements method is developed to solve the considered boundary value problems. The results obtained using this method are compared with results obtained using an analytical approach and good agreement is shown. Also, previous work in the area has obtained the flow velocity components using numerical approximations, [3]. In this work the velocity components are found using integration and these integrals determined numerically and analytically. Analytical formulas for all the integrals in the expressions for the stream function, vorticity and ${ }_{225}$ Cartesian velocity components are also given in this work. From the results it is shown that using analytical integrals in the BEM procedure reduces computing time and also that the BEM method gives accurate results. Various situations have been considered and the method has been shown to perform well. Hence it has been demonstrated that the approach outlined here, with the analytical derivation of the integrals within the BEM, is an accurate and efficient method to model filtration problems.

The developed method was also tested by the solution of the problems of fluid flow through the porous cylinder in rectangular domain and the fluid flow between eccentric cylinders. High accuracy of the method was confirmed.

The present approach will be applied in future work to calculate the dusty air flow in an array of filter fibers taking into account the change of the fiber shape and size due to the dust particle deposition.

The work was supported by the RFBR (grant N15-01-06135) and EPSRC (travel grant EP/M003841/1).

\section{References}

[1] V. Kirsh, Stokes flow past periodic rows of porous cylinders, Theoretical Foundations of Chemical Engineering 40 (5) (2006) 465-471. doi:10.1134/S0040579506050034.

URL http://dx.doi.org/10.1134/S0040579506050034

$[2] \mathrm{S}$.

Zaripov,

O. Soloveva,

S. Solovev, 
Inertial deposition of aerosol particles in a periodic row of porous cylinders,

Aerosol Science and Technology 49 (2015) 400-408.

doi:10.1080/02786826.2015.1036834,

URL http://www .tandfonline.com/doi/full/10.1080/02786826.2015.1036834

250 [3] S. Dunnett, C. Clement, A numerical model of fibrous filters containing deposit,

Engineering Analysis with Boundary Elements 33 (5) (2009) 601-610.

doi:http://dx.doi.org/10.1016/j.enganabound.2008.10.010.

URL/http://www.sciencedirect.com/science/article/pii/S0955799708001963

[4] S. Kuwabara, The forces experienced by randomly distributed parallel circular cylinders or spheres in a viscous flow at small reynolds numbers, Journal of Physical Society of Japan 14 (4) (1959) 527 - 532.

[5] I. Stechkina, Drag of porous cylinders in a viscous fluid at low reynolds numbers, Fluid Dyn. 14 (6) (1979) 912-915.

[6] H. Marshall, M. Sahraoui, Kaviany,

An improved analytic solution for analysis of particle trajectories in fibrous, two-dimensional filters,

Physics of Fluids $6 \quad$ (2) (1994) 507-520.

doi:http://dx.doi.org/10.1063/1.868346.

URL http://scitation.aip.org/content/aip/journal/pof2/6/2/10.1063/1.868346

[7] G. S. Beavers, D. D. Joseph, Boundary conditions at a naturally permeable wall,

Journal of Fluid Mechanics $30 \quad$ (1967) 197-207.
doi:10.1017/S0022112067001375.

URL http://journals . cambridge.org/article_S0022112067001375

[8] D. A. Nield, A. Bejan, Convection in Porous Media, Springer-Verlag, New York, 1992.

[9] M. Jaswon, G. Symm, Integral equation methods in potential theory and electrostatics, Academic Press, New York, 1977.

[10] M. Kelmanson, An integral equation method for the solution of singular slow flow problems, Journal of Computational Physics $51 \quad$ (1) (1983) 139-158. 
doi:http://dx.doi.org/10.1016/0021-9991(83)90084-0.

[11] M. Kelmanson, A boundary integral equation method for the study of slow flow in bearings with arbitrary geometries, Journal of Tribology 106 (2) (1984) 260-264. doi:10.1115/1.3260897.

[12] J.-T. Chen, C.-C. Hsiao, S.-Y. Leu, A new method for stokes problems with circular boundaries using degenerate kernel and fourier series, International Journal for Numerical Methods in Engineering 74 (13) (2008) 1955-1987. doi:10.1002/nme.2240 